\title{
Laparoscopic Peritoneal Dialysis Catheter Insertion
}

\section{Saud Faleh Alomani*, Abdulrahaman Alballa, Ayed Almutairi, Nouf Yaseen and Taghreed Alajajee}

Prince Sultan Military Medical City, Department of Surgery, Riyadh, Kingdom of

Saudi Arabia

*Corresponding Author: Saud Faleh Alomani, Prince Sultan Military Medical

City, Department of Surgery, Riyadh, Kingdom of Saudi Arabia.

DOI: 10.31080/ASWH.2020.02.0084
Received: December 24, 2019

Published: January 29, 2020

(C) All rights are reserved by Saud Faleh

Alomani., et al.

\begin{abstract}
Objective: To assess laparoscopic peritoneal dialysis (PD) catheter placement techniques and complications among End Stage Renal Disease (ESRD) patients at Prince Sultan Military Medical City (PSMMC), Riyadh, Kingdom of Saudi Arabia.

Methods: Retrospective review was performed on patients who underwent laparoscopic PD catheter placement with tunneling +/- adhesionlysis and omentopexy or omentectomy, between July 2008 and June 2018, by a single surgeon at Prince Sultan Military Medical City (PSMMC), Riyadh, Kingdom of Saudi Arabia.

Result: Two hundred and twenty-two patients underwent laparoscopic PD catheter insertion during the study period. Late complications encountered include: tuberculosis (TB) peritonitis in 9 patients (4\%), catheter dysfunction in 13 patients (6\%), adhesions in 2 patients (1\%), bacterial peritonitis in 25 patients (11\%), leakage in 15 patients (7\%), and 16 patients (7\%) developed an umbilical hernia. There is a superficial fungal infection around the catheter exit in one patient $(0.45 \%)$, and exit-site bacterial infection in 7 patients (3\%). Catheter remained active for the first insertion in 33 patients $(15 \%)$ and was reinserted in 7 patients (3\%). Fifty-one patients (23\%) underwent renal transplantation, and $82(37 \%)$ patients were switched to hemodialysis. Eleven patients (5\%) were lost to follow up, and 45 patients (20\%) died due to their chronic illness. Seven (28\%) of the 25 patients with bacterial peritonitis were cured with conservative management in the form of antibiotics and other conservative measures. There was no procedurerelated mortality or bleeding.

Conclusion: Laparoscopic PD catheter insertion is safe and effective. Utilization of this technique provides a low rate of PD catheter complications. However, we encountered a noticeable rate of peritoneal tuberculosis in our patients.

Keywords: Peritoneal Dialysis Catheter; Laparoscopic Surgery; Complications of Peritoneal Catheter Insertion
\end{abstract}

\section{Introduction}

Tenckhof and Schechter described a percutaneous nonvisualized method of peritoneal dialysis catheter (PDC) placement in 1968 [1]; however, this was associated with a risk of bowel and vessel injury, as well as a high incidence of malpositioned PDCs resulting in failure rates of up to $65 \%$ at two years. Subsequently, the procedure was modified to open placement of the PDC tip into the pelvis under direct surgical vision via minilaparotomy [2]. A peritoneoscopic technique was later used in 1981 by Ash., et al. [3] to improve catheter function and decrease complications. Over the last decade, several reports have described laparoscopic placement of PDCs, [4-11] facilitating direct visualization of the peritoneal cavity and exact placement of the catheter tip deep into the cul-de-sac. Currently, $4 \%$ of patients requiring peritoneal dialysis (PD) in Saudi Arabia are served at Prince Sultan Military Medical City (PSMMC). We have 54 patients on peritoneal dialysis out of the 1372 registers in Saudi Center of Organ Transplant (SCOT) as re- ported in 2016. Peritoneal dialysis is gaining popularity among our end-stage renal disease (ESRD) patients at PSMMC, and over the last few years, based on our hospital nephrology registry; this has grown to approximately $16 \%$ of the total number of patients who are on dialysis. This modality of treatment is in demand globally, reaching overall of $9 \%$ of patients on dialysis in the Kingdom, based on SCOT data published in 2016. The advantages of PD over hemodialysis include the preservation of vascular access sites, reduced risk of transmission of blood-borne infection, and possibly better preservation of residual renal function [13]. Other advantages are lower cost, increased patient mobility, better control of hypertension, maintenance of blood chemistries, no requirement for anticoagulants, and no routine blood loss [4]. However, the disadvantages and associated complications include peritonitis, pericatheter infection, catheter malfunction, dialysate leaks, sclerosing peritonitis, and hernias [14]. In this study, we assessed the laparoscopic PDC placement techniques and complications among ESRD patients at PSMMC over ten years duration. 


\section{Methods}

We performed a retrospective review of 222 patients who underwent a laparoscopic PDC placement procedure over a 10-year period from July 2008 to June 2018 at PSMMC, Riyadh, Saudi Arabia. All patients were managed by a single surgical team and followed regularly by one clinical nurse specialist (RN).

\section{Surgical technique}

The exit-site was chosen preoperatively by marking the abdomen wall on the side opposite to the dominant hand, and the patients were placed on the operating room table on a supine position. All procedures were carried out under general anesthesia. One dose of prophylactic antibiotics (Ciprofloxacin) was administered preoperatively, and 2 doses postoperatively. Iodine povidone was applied 3 times to the abdomen, followed by draping of the area. A supraumbilical open technique (modified Hasson technique) was used to establish a pneumoperitoneum of $14 \mathrm{~mm} \mathrm{Hg}$. We used a $5 \mathrm{~mm}$ trocar and zero-degree laparoscope. After visualization of the abdominal cavity, a $10 \mathrm{~mm}$ trocar was inserted in the right upper abdomen at the mid- clavicular line, 5-6 cm below the costal margin, and the patient was re positioned with head down and table tilted to the left side. Once the pelvis was free from adhesions and adhesiolysis was completed, if needed, the entrance and exit-sites were marked. We found it helpful to lay the catheter on the abdomen to estimate the entrance site based on the length of the patient's torso. The tip should easily reach the cul-de-sac, and the top of the curl should be at the pubic symphysis. We preferred an entrance site within the linea alba, $2 \mathrm{~cm}$ below the umbilicus. The PDC or Tenckhoff set is composed of a catheter, introducer needle, 10 cc syringe, guidewire, \#11 scalpel, 16-French sheath, dilator, tunneling stylet, 6 gauze sponges, clamp, and cap (Figure 1 and Figure 2). The dissection (tunneling extend caudally extraperitoneal to a point just above the dome of the urinary bladder. Tunneling is carried out with Lahey forceps for a length of 7-10 $\mathrm{cm}$ along the midline without breaching the peritoneum under direct visualization. The peritoneum is then opened at this point. The catheter is inserted from the $10 \mathrm{~mm}$ port and then pulled out using the Lahey forceps leaving the coil fenestrated catheter end within the pouch of Douglas. The caudal cuff was held to the peri peritoneum (extra-peritoneal), and the outer cuff tunneled to an exit-site 30-40 degrees from a horizontal imaginary line at the level of the umbilicus, in the subcutaneous tissue. Omentopexy is done if needed and the abdomen is then deflated, and the trocars removed. The fascia of the $10 \mathrm{~mm}$ trocar was closed with non-absorbable sutures and skin closed with skin clip. The catheter was tested on table for any dysfunction or leakage with 1-2 liters of dialysate solution and then evacuated. Five thousand units of heparin diluted in $50 \mathrm{ml}$ saline and injected into the catheter. The catheter was used after 2 to 3 weeks later. All patients are followed up in the PD catheter outpatient's clinic.

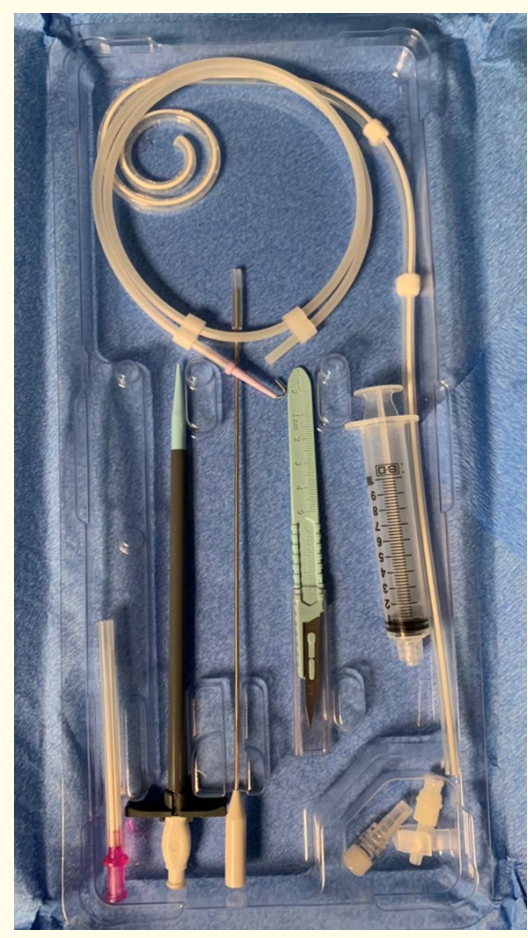

Figure 1: Coiled Peritoneal Dialysis catheter.

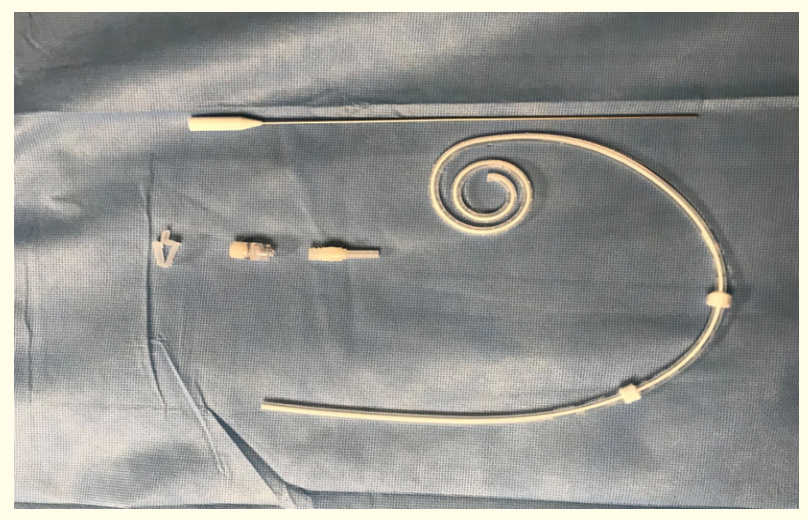

Figure 2: Coiled Peritoneal Dialysis Catheter

\section{Results}

Two hundred and twenty-two patients underwent laparoscopic PDC insertion under general anesthesia between July 2008 and June 2018. All patients were discharged on the first or second postoperative day. Combined diabetes and hypertension were the cause of renal failure in a large percentage $(47 \%)$ of our study population (Table 1). Hypertension was documented in 47 patients (21\%), diabetes in 42 patients (19\%), glomerulonephritis in 13 patients $(6 \%)$, renal artery stenosis in 7 patients $(3 \%)$, polycystic kidney disease in 5 patients (2\%), and systemic lupus erythematosus in 4 patients (2\%). No visceral or vascular injuries occurred. Twenty-five patients had peritonitis (Table 2); 7 were cured with medical treatment and intravenous antibiotics (PD catheter was 
not removed) and 4 patients died ( 3 sepsis, and one myocardial infarction). 14 patients out of the 25 patients have been shifted to hemodialysis and their PD catheter was removed. A total of eightytwo patients was switched to hemodialysis (Table 3). Nine patients had peritoneal tuberculosis, of which 8 shifted to hemodialysis, and 3 patients died; 2 because of the hepato-renal syndrome, and one because of a stroke. One patient with peritoneal tuberculosis still had an active PD catheter after completion of TB treatment course. Fifteen patients had early leakage and underwent a second operation for the closure of the peritoneal opening. Thirteen patients had late catheter malfunctions, of which, 7 , required reinsertion. Two patients had adhesions, both requiring adhesiolysis and re-insertion. Fungal infection occurred in one patient, which was cured by antifungal treatment. Sixteen patients had an umbilical hernia requiring open umbilical repair. 13 patients have been shifted to hemodialysis up on their requests (11 patients of the lost follow up with us). At the end of the study period, 33 (15\%) patients had active PDCs, 51(23\%) patients had been transplanted, and $82(37 \%)$ patients were switched to hemodialysis (Table 4 ).

\begin{tabular}{|l|c|c|}
\hline \multicolumn{1}{|c|}{ Causes } & Number of patients & \% \\
\hline $\begin{array}{l}\text { Diabetes Meletus and } \\
\text { Hypertension }\end{array}$ & 104 & $(47)$ \\
\hline Hypertension & 47 & $(21)$ \\
\hline Diabetes Meletus & 42 & $(19)$ \\
\hline Glomerulonephritis & 13 & $(6)$ \\
\hline Renal Artery Stenosis & 7 & $(3)$ \\
\hline Polycystic Kidney & 5 & $(2)$ \\
\hline Systemic Lupus & 4 & $(2)$ \\
\hline
\end{tabular}

Table 1: Causes of renal failure in 222 patients underwent Laparoscopic Peritoneal dialysis Catheter Insertion.

\begin{tabular}{|l|c|c|}
\hline \multicolumn{1}{|c|}{ Complications } & Numbers of patients & \% \\
\hline Peritoneal Tuberculosis & 9 & $(4)$ \\
\hline Dialysate leakage & 15 & $(7)$ \\
\hline Catheter malfunction & 13 & $(6)$ \\
\hline Late adhesions & 2 & $(1)$ \\
\hline Fungal infection & 1 & $(0.45)$ \\
\hline Hernia & 16 & $(7)$ \\
\hline Peritonitis & 25 & $(11)$ \\
\hline
\end{tabular}

Table 2: Complications encountered.

\begin{tabular}{|l|c|c|}
\hline Reasons & Numbers of patients & \% \\
\hline Leakage & 15 & $(18)$ \\
\hline Catheter malfunction & 13 & $(16)$ \\
\hline T.B Peritonitis & 8 & $(10)$ \\
\hline Late adhesions & 2 & $(2)$ \\
\hline Fungal infections & 1 & $(1)$ \\
\hline Hernia & 16 & $(20)$ \\
\hline Bacterial infections & 14 & $(17)$ \\
\hline On patients request & 13 & $(16)$ \\
\hline
\end{tabular}

Table 3: Reasons to shift to Hemodialysis (82 patients).

\begin{tabular}{|l|c|c|}
\hline \multicolumn{1}{|c|}{ Catheter disposition } & Numbers of patients & \% \\
\hline Active & 33 & $(15)$ \\
\hline Transplanted & 51 & $(23)$ \\
\hline Shifted to Hemodialysis & 82 & $(37)$ \\
\hline Lost follow up & 11 & $(5)$ \\
\hline Died & 45 & $(20)$ \\
\hline
\end{tabular}

Table 4: Catheter disposition in 222 patients.

\section{Discussion}

Laparoscopic insertion of PDCs is an innovative use of minimally invasive surgery. Our technique was successful as there was no need to convert to the open method for insertion or re-insertion of the PDC's. The designation of the exit-site of the catheter was comfortable for the patient because of the cultural type of dress worn.

A meta-analysis by Strippoli., et al. [15] identified 17 eligible trials with 1098 patients. The trials included 8 straight versus coiled catheters, one of single versus double cuff catheters, and one of immobilizer devices. There were no significant differences with laparoscopy compared to laparotomy for peritonitis, exit site/tunnel infection, or catheter removal/replacement. Standard insertion with no subcutaneous burying of the catheter versus implantation and subcutaneous burying was not associated with a significant reduction in peritonitis rate, exit-site/tunnel infection rates, or overall mortality. Midline compared to lateral insertion showed no significant difference in the risk of peritonitis or exit-site/tunnel infection. There was no significant difference in the risk of peritonitis, or its rate, exit-site/tunnel infection, or its rate, or catheter removal/replacement between straight versus coiled catheters. One trial compared single versus double cuffed catheters and showed no significant difference in the risk of peritonitis, exit-site/tunnel infection, or catheter removal/replacement. One trial compared immobilization versus no immobilization of PDC and showed no significant difference in the risk of peritonitis and exit-site/tunnel infection.

Abdominal wall hernia is a known complication in patients undergoing peritoneal dialysis, occurring in up to $12 \%$. Peritoneal tuberculosis is another known complication of PCD [16-18]. However, we found a surprisingly high rate among our patients. Most probably because this area is endemic for tuberculosis, in addition to the low cellular type of immunity in renal failure patients $[19,20]$. The prevalence and incidence of ESRD and the requirement for Renal Replacement Therapy (RRT) are increasing in Saudi Arabia (SCOT report 2016). With the scarcity of available organ for transplant, patients must choose between hemodialysis (HD) and peritoneal dialysis (PD). Currently, most of our patients are on HD and only $16 \%$ are in PD. With the advantages of laparoscopic 
surgery, this may encourage the patients to choose the option of peritoneal dialysis.

In conclusion, laparoscopic PDC insertion is safe and effective. The utilization of this technique provides a low rate of PDC complications. However, we did encounter a noticeable rate of peritoneal tuberculosis in our study group.

\section{Conclusion}

Laparoscopic PD catheter insertion is safe and effective. The utilization of this technique provides a low rate of PD catheter complications. However, we encountered a noticeable rate of peritoneal tuberculosis in our patients.

\section{Bibliography}

1. Tenckhoff $\mathrm{H}$ and Schechter H. "A bacteriologically safe peritoneal access device". Transactions - American Society for Artificial Internal Organs 14 (1968): 181-187.

2. Nghiem DD. "A technique of catheter insertion for uncomplicated peritoneal dialysis". Surgery, Gynecology and Obstetrics 157 (1983): 573-576.

3. Brandt CP and Franceschi D. "Laparoscopic placement of peritoneal dialysis catheters in patients who have undergone prior abdominal operations". Journal of the American College of Surgeons 178 (1994): 515-516.

4. Watson DI., et al. "Secure placement of peritoneal dialysis catheters using a laparoscopic technique". Surgical Laparoscopy Endoscopy 6 (1996): 35-37.

5. Nijhu is PH., et al. "Laparoscopic introduction of a continuous peritoneal dialysis (capd) catheter by a two-puncture technique". Surgical Endoscopy 10 (1996): 676 -679.

6. Crabtree JH and Fishma nA. "Yideolaparoscopic implantation of peritoneal dialysis catheters". Surgical Endoscopy 13 (1999): 186-190.

7. Barone GW., et al. "Technique for laparoscopy-assisted complicated peritoneal dialysis catheter placement". Journal of Laparoendoscopic and Advanced Surgical Techniques 12 (2002): 53-55.

8. Stephe H., et al. "Guidelines for laparoscopic dialysis access surgery”. Surgical Endoscopy 28 (2014): 3016-3045.

9. Lu CT., et al. "Laparoscopic placement of peritoneal dialysis catheters: 7 years experience". ANZ Journal of Surgery 73 (2003): 109-111.

10. Manouras AJ., et al. "Laparoscopic placement of Oreopoulos Zellerman catheters in CAPD patients". Peril Dial J 24 (2004): 252-255.
11. Crabtree JH and Fishman A. "A laparoscopic method for optimal peritoneal dialysis access". American Surgery 71 (2005): $135-143$

12. Harissis HV., et al. "A new simplified one port laparoscopic technique of peritoneal dialysis catheter placement with intraabdominal fixation". American Journal of Surgery 192 (2006): 125-129.

13. Saudi Center for Organ Transplantation. Scot data". Saudi Journal of Kidney Disease and Transplantation 8 (1997): 320-328.

14. Ellam $\mathrm{T}$ and Wilkie M. "Peritoneal dialysis". Medicine 35 (2007): 466-469.

15. Olcott C., et al. "Continuous ambulatory peritoneal dialysis. Technique of catheter insertion and management of associated surgical complications". American Journal of Surgery 146 (1983): 98-102.

16. Strippoli GF., et al. "Catheter type, placement and insertion techniques for preventing peritonitis in peritoneal dialysis patients". Cochrane Database System Review (2004): CD004680.

17. Bullmaster JR., et al. "Surgical aspects of the Tenckhoff peritoneal dialysis catheter. A 7-year experience". American Journal of Surgery 149 (1985): 339-342.

18. Lui SL., et al. "Tuberculosis infection in Chinese patients undergoing continuous ambulatory peritoneal dialysis". American Journal of Kidney Diseases 38 (2001): 1055-1060.

19. Lui SL., et al. "Optimal treatment and long-term outcome of tuberculous peritonitis complicating continuous ambulatory peritoneal dialysis". American Journal of Kidney Diseases 28 (1996): 747-751.

20. Pesanti EL. "Immunologic defects and vaccination in patients with chronic renal failure". Infectious Disease Clinics of North America 15 (2001): 813-832.

\section{Assets from publication with us}

- Prompt Acknowledgement after receiving the article

- Thorough Double blinded peer review

- Rapid Publication

- Issue of Publication Certificate

- High visibility of your Published work

Website: https://www.actascientific.com/

Submit Article: https://www.actascientific.com/submission.php Email us: editor@actascientific.com

Contact us: +919182824667 